\title{
On the dynamics of StemBells: Microbubble-conjugated stem cells for ultrasound- controlled delivery
}

Tom J. A. Kokhuis, Benno A. Naaijkens, Lynda J. M. Juffermans, Otto Kamp, Antonius F. W. van der Steen, Michel Versluis, and Nico de Jong

Citation: Appl. Phys. Lett. 111, 023701 (2017); doi: 10.1063/1.4993172

View online: https://doi.org/10.1063/1.4993172

View Table of Contents: http://aip.scitation.org/toc/apl/111/2

Published by the American Institute of Physics

\section{Articles you may be interested in}

Announcement: Applied Physics Letters eliminates publication fees, effective 1 June 2017

Applied Physics Letters 111, 010201 (2017); 10.1063/1.4989855

Peculiar torsion dynamical response of spider dragline silk

Applied Physics Letters 111, 013701 (2017); 10.1063/1.4990676

Acoustic force measurements on polymer-coated microbubbles in a microfluidic device

The Journal of the Acoustical Society of America 141, 3364 (2017); 10.1121/1.4979933

Enhanced sensing of gas molecules by a 99.9\% semiconducting carbon nanotube-based field-effect transistor sensor

Applied Physics Letters 111, 022102 (2017); 10.1063/1.4991970

Cold nanoindentation of germanium

Applied Physics Letters 111, 021901 (2017); 10.1063/1.4993163

Plasmonic metasurface for optical rotation

Applied Physics Letters 111, 023102 (2017); 10.1063/1.4993429

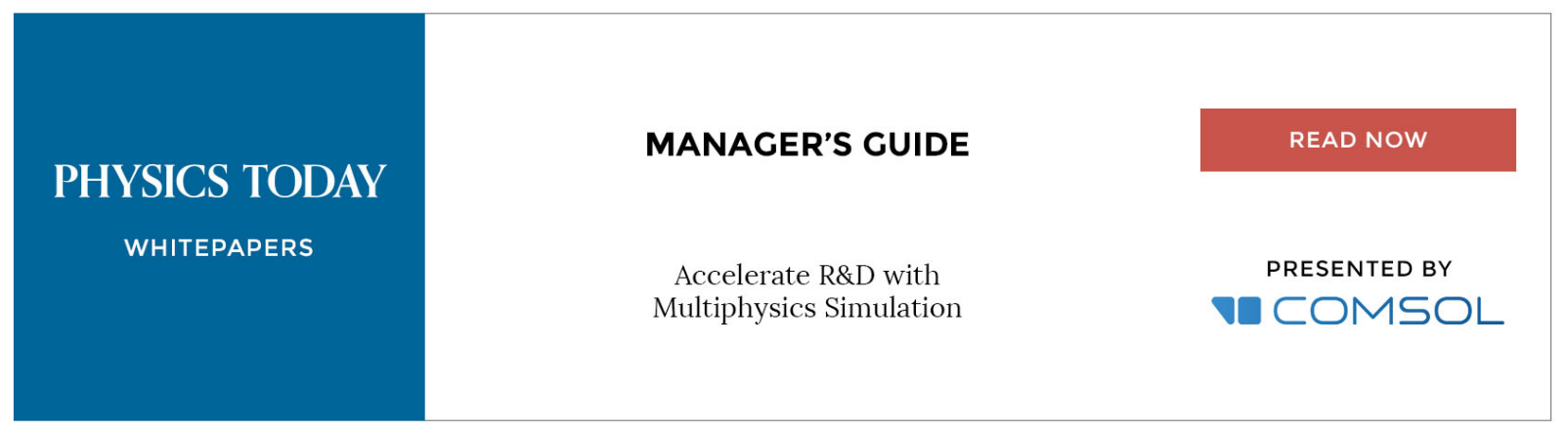




\title{
On the dynamics of StemBells: Microbubble-conjugated stem cells for ultrasound-controlled delivery
}

\author{
Tom J. A. Kokhuis, ${ }^{1,2, a)}$ Benno A. Naaijkens, ${ }^{2,3}$ Lynda J. M. Juffermans, ${ }^{2,4}$ Otto Kamp, ${ }^{2,5}$ \\ Antonius F. W. van der Steen, ${ }^{1,2,6,7}$ Michel Versluis, ${ }^{8,9}$ and Nico de Jong ${ }^{1,2,6}$ \\ ${ }^{1}$ Biomedical Engineering, Erasmus Medical Center, 3015 CN Rotterdam, The Netherlands \\ ${ }^{2}$ Netherlands Heart Institute, 3511 EP Utrecht, The Netherlands \\ ${ }^{3}$ Pathology, VU University Medical Center, 1081 HV Amsterdam, The Netherlands \\ ${ }^{4}$ Physiology, VU University Medical Center, 1081 HV Amsterdam, The Netherlands \\ ${ }^{5}$ Cardiology, VU University Medical Center, 1081 HV Amsterdam, The Netherlands \\ ${ }^{6}$ Acoustical Wavefield Imaging, Delft University of Technology, 2600 AA Delft, The Netherlands \\ ${ }^{7}$ Shenzhen Institutes of Advanced Technology, 518055 Shenzhen, China \\ ${ }^{8}$ Physics of Fluids Group, University of Twente, 7500 AE Enschede, The Netherlands \\ ${ }^{9}$ MIRA Institute of Biomedical Technology and Technical Medicine, University of Twente, 7522 NB Enschede, \\ The Netherlands
}

(Received 7 April 2017; accepted 27 June 2017; published online 11 July 2017)

\begin{abstract}
The use of stem cells for regenerative tissue repair is promising but hampered by the low number of cells delivered to the site of injury. To increase the delivery, we propose a technique in which stem cells are linked to functionalized microbubbles, creating echogenic complex dubbed StemBells. StemBells are highly susceptible to acoustic radiation force which can be employed after injection to push the StemBells locally to the treatment site. To optimally benefit from the delivery technique, a thorough characterization of the dynamics of StemBells during ultrasound exposure is needed. Using high-speed optical imaging, we study the dynamics of StemBells as a function of the applied frequency from which resonance curves were constructed. A theoretical model, based on a modified Rayleigh-Plesset type equation, captured the experimental resonance characteristics and radial dynamics in detail. Published by AIP Publishing.

[http://dx.doi.org/10.1063/1.4993172]
\end{abstract}

Stem cell therapy holds the potential to regenerate damaged cardiac tissue after a myocardial infarction. ${ }^{1}$ However, the low retention and engraftment rate of transplanted cells within the infarcted area are currently hampering successful myocardial regeneration. ${ }^{2}$ Typically, only a few percent of the transplanted cells are retained in the infarcted area, even after intracoronary administration, with the majority of the cells ending up in the spleen and liver. ${ }^{3}$ The reduction of the infarct size and long-term improvement in cardiac functioning have been shown to correlate with the amount of engrafted cells, ${ }^{4}$ and thus, an efficient method facilitating enhanced and localized stem cell delivery is needed.

We propose a technique for localized stem cell delivery using targeted microbubble ultrasound contrast agents and acoustic radiation force. ${ }^{5,6}$ Application of acoustic radiation force can be used to promote the delivery and adherence of targeted microbubbles in vivo. ${ }^{7-9}$ By decorating stem cells with targeted microbubbles, acoustically active complexes named StemBells are created, which can be propelled locally toward and arrested at the vessel wall using acoustic radiation forces.

The effects of radiation force on microbubbles are maximum at the resonance frequency, where the volumetric oscillations are maximum. ${ }^{10,11}$ Complex interactions between microbubbles in close proximity can affect the oscillatory dynamics, ${ }^{12}$ presumably due to a shift in the resonance frequency of the microbubbles. In order to optimize the propulsion, an acoustical characterization of the StemBells is

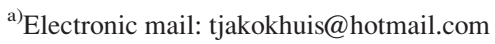

needed. The aim of this study was therefore to characterize the StemBell dynamics in response to ultrasound waves using high-speed optical imaging.

Cells from the stromal vascular fraction of human adipose tissue were cultured in a platelet lysate-supplemented medium. ${ }^{13}$ Cells from passages 2-5 were used for the experiments; the median diameter of the cells was $18 \mu \mathrm{m}$. Biotinylated lipidcoated microbubbles with a perfluorobutane $\left(\mathrm{C}_{4} \mathrm{~F}_{10}\right)$ gas core were made by sonication as described before. ${ }^{14,15}$ The median diameter of the microbubbles was $3 \mu \mathrm{m}$. Microbubbles were labeled with the lipophilic fluorescent dye octadecylindocarbocyanine (DiI, Molecular Probes, Life Technologies Europe, Bleiswijk, the Netherlands). Monoclonal antibodies against CD90 (BD Biosciences, Breda, the Netherlands), a cell surface protein constitutionally expressed by the stem cells, were conjugated to the microbubbles using biotin-avidin bridging. ${ }^{16}$ Cells were fluorescently labeled with CellTracker Green CMFDA (Molecular Probes), trypsinized, washed, and mixed with CD90-functionalized microbubbles by continuous rotation at $2 \mathrm{rpm}$ (Rotator PTR-35, Grant-bio, Shepreth, UK) for $30 \mathrm{~min}$ at room temperature to create the microbubble-conjugated stem cells. Dependent on the mixing ratio (stem cell: microbubble), different saturation grades can be obtained. ${ }^{5}$ Here, we mainly focussed on the dynamics of saturated StemBells ( $>30$ bubbles/ cell) as those were found to be most susceptible to acoustic forcing in vivo. ${ }^{5}$ StemBells were insonified at different pressures $\left(\mathrm{P}_{-}=50-200 \mathrm{kPa}\right)$ with a Gaussian tapered ultrasound pulse (4-8 cycles) at driving frequencies between 0.4 and $3.0 \mathrm{MHz}$. The pulses were generated with an arbitrary waveform generator (Tabor Electronics Ltd., Model 8026, Tel Hanan, Israel) and 
amplified by using a $60 \mathrm{~dB}$ RF linear amplifier (ENI, Model A500, Rochester, NY, USA). A broadband polyvinylidene fluoride (PVDF) transducer (PA275, Precision Acoustics, Dorchester, UK) was used between 1 and $3 \mathrm{MHz}$; a ceramic transducer (V318, Panametrics, Inc., Waltham, MA, USA) with a center frequency of $500 \mathrm{kHz}$ was used for frequencies below $1 \mathrm{MHz}$. All experiments were performed at $37^{\circ} \mathrm{C}$. The dynamics of isolated StemBells was imaged with a customized BXFM microscope (Olympus Nederland B.V., Zoeterwoude, the Netherlands) using a $40 \times$ water-immersion objective lens (LUMPLFL, Olympus, numerical aperture 0.8) in combination with a $2 \times$ magnification ring. The optical focus and acoustical focus were coaligned prior to each experiment. Images were relayed to the Brandaris128 ultra-fast framing camera. ${ }^{17,18}$

Figure 1 shows brightfield (a), green fluorescent (b) [showing the cell], and red fluorescent (c) [showing the surrounding microbubbles] images of a StemBell saturated with targeted microbubbles. Saturated StemBells were observed to vibrate as a single entity (Multimedia view in the supplementary material). To quantify the response to an ultrasound burst, the StemBell contour was determined using custom software based on a minimum cost algorithm [see the red line Fig. 1(a)]. The radius of a StemBell was defined as the radius of the circle with an area equivalent to the area enclosed by this contour [see the yellow line Fig. 1(a)]. Using this contour detection technique, the experimental radius-time curve, $R(t)$, was determined. Figure 1(d) shows the $R(t)$-curve of the StemBell in response to an 8-cycle ultrasound burst at a frequency of $1.2 \mathrm{MHz}$ and a driving pressure $P_{-}$of $=200 \mathrm{kPa}$. The discrete Fourier transform of each $R(t)$-curve was then calculated to determine the fundamental vibration amplitude $\left(A_{1}\right)$ of the StemBell for each driving frequency, from which its resonance curve was constructed. ${ }^{19}$ To obtain the experimental resonance frequency, the data points were fitted with the general amplitude response of a harmonic oscillator, which reads ${ }^{20}$
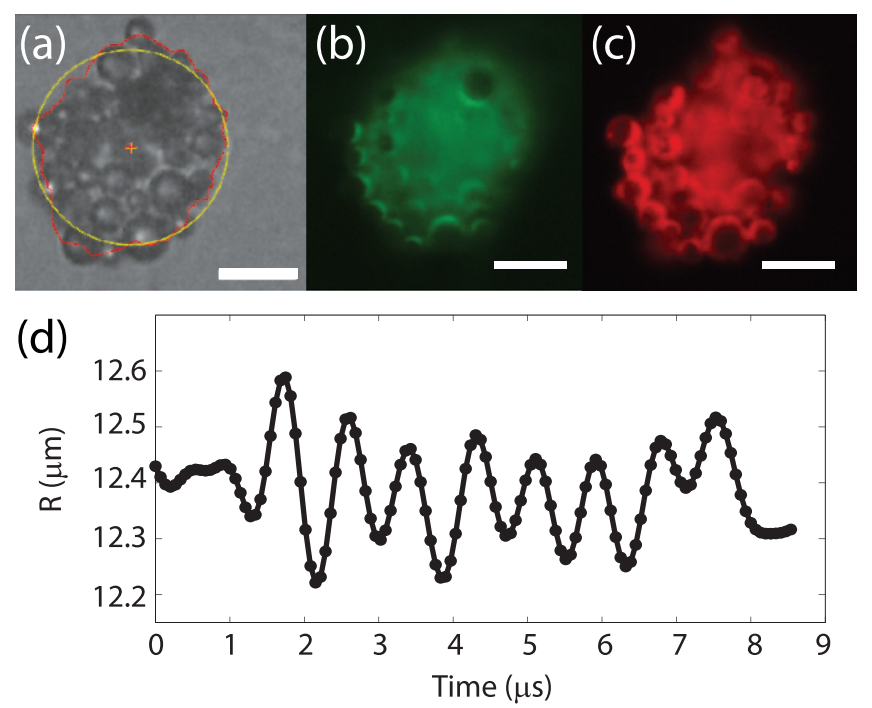

FIG. 1. (a) Brightfield, (b) green fluorescent (indicating the stem cell), and (c) red fluorescent (indicating surrounding microbubbles) images of a saturated StemBell. The radius of a StemBell was defined as the radius of the circle [yellow line in panel (a)] with the area equivalent to the area enclosed by the contour of the StemBells [red line in panel (a)]. The scale bar corresponds to $10 \mu \mathrm{m}$. (d) Radius-time curve of the StemBell in response to an 8 cycle ultrasound burst at $1.2 \mathrm{MHz}$ and $P_{-}=200 \mathrm{kPa}$.

$$
\operatorname{Re}(f)=\frac{R e_{0}}{\left(1-f^{2} / f_{0}^{2}\right)^{2}+\left(\delta f / f_{0}\right)^{2}},
$$

where $f$ is the driving frequency and $f_{0}$ (eigenfrequency), $\delta$ (damping coefficient), and $R e_{0}$ (amplitude) are the fitting parameters. The uncertainty in the obtained resonance frequency was estimated to be $\sim 8 \%$.

Figure 2(a) shows the relative fundamental vibration amplitude, $A_{1} / R_{0}$, as a function of the driving frequency between 1 and $3 \mathrm{MHz}$ for a saturated StemBell (red dots). For comparison, the response of a single isolated adherent microbubble attached to a stem cell (blue squares) is also shown. The resting radius of the adherent bubble is $2.8 \mu \mathrm{m}$, which is equal to the mean volume-weighted radius of the microbubbles surrounding the saturated StemBell. The vibrational amplitude of the single bubble is maximal at a frequency of 1.3 MHz. However, in the case of the StemBell, only the falling edge of the resonance curve is captured, implying a resonance frequency $<1 \mathrm{MHz}$. Figure 2(b) shows the response of a saturated StemBell $\left(R_{0}=10 \mu \mathrm{m}\right)$ between 0.4 and $0.8 \mathrm{MHz}$ (limited by the bandwidth of the transducer). The StemBell is resonantly excited at a driving frequency of $0.6 \mathrm{MHz}$, much lower (i.e., >50\%) than the individual resonance behavior of the bubbles around the cell.

For bubble clouds, it has been shown that the total gas volume or void fraction, and not the size of the individual bubbles within the cloud, dictates the resonance frequency. ${ }^{21}$ Therefore, to corroborate our experimental findings, numerical simulations were performed using a modified RayleighPlesset equation incorporating the total gas volume of the microbubble layer. The StemBell was modeled as a liquid globule (with radius $R_{c}$ ), surrounded by a thin gas layer of thickness $d_{0}$. The central liquid core can be regarded incompressible compared to the compressibility of the surrounding gas layer. It was therefore assumed that, in response to an external pressure variation $P(t)$, only the radius $R$ of the external gas/fluid interface will change, varying the volume $V$ of the surrounding gas layer. The radius of the liquid core $R_{c}$ was considered to be constant. Furthermore, similar to coated microbubbles, ${ }^{22}$ the gas layer was assumed to be coated with a viscoelastic lipid shell, with an effective shell elasticity $\chi$ and an effective shell viscosity $\kappa_{s}$. For such a
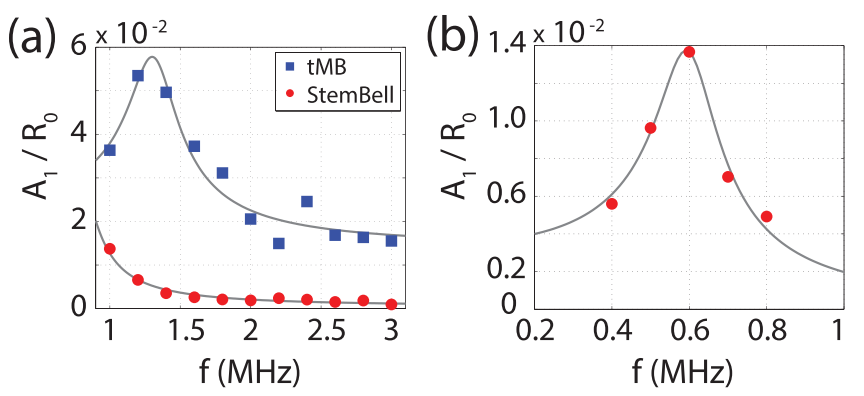

FIG. 2. (a) Relative fundamental vibration amplitude as a function of driving frequency for an adherent microbubble with the resting radius $R_{0}=2.8 \mu \mathrm{m}$ (blue squares) and a saturated StemBell with the resting radius $R_{0}=12.6 \mu \mathrm{m}$ (red dots). The amplitude of the driving pressure was $200 \mathrm{kPa}$. (b) Relative fundamental vibration amplitude showing the fundamental amplitude response of a StemBell with an equivalent radius of $10 \mu \mathrm{m}$ to driving frequencies from 0.4 to $0.8 \mathrm{MHz}$ at $P_{-}=50 \mathrm{kPa}$. 
TABLE I. Notation.

\begin{tabular}{ll}
\hline \hline Symbol & \multicolumn{1}{c}{ Description } \\
\hline$\rho_{1}$ & Liquid density \\
$R$ & Outer radius \\
$R_{0}$ & Resting radius outer wall \\
$\dot{R}$ & Radial velocity outer wall \\
$\ddot{R}$ & Radial acceleration outer wall \\
$R_{\mathrm{c}}$ & Radius liquid globule \\
$d_{0}$ & Resting thickness gas layer \\
$P_{0}$ & Ambient pressure \\
$P(t)$ & Acoustic driving pressure \\
$\sigma(R)$ & Surface tension outer interface \\
$\sigma\left(R_{0}\right)$ & Initial surface tension outer interface \\
$\gamma$ & Polytropic gas exponent \\
$\mu$ & Dynamic liquid viscosity surrounding liquid \\
$\kappa_{\mathrm{s}}$ & Effective shell viscosity \\
$\chi$ & Effective shell elasticity \\
\hline \hline
\end{tabular}

system, a modified Rayleigh-Plesset equation can be defined, which reads

$$
\begin{aligned}
\rho_{l}\left(R \ddot{R}+\frac{3}{2} \dot{R}^{2}\right)= & \left(P_{0}+\frac{2 \sigma\left(R_{0}\right)}{R_{0}}\right)\left(\frac{R_{0}^{3}-R_{c}^{3}}{R^{3}-R_{c}^{3}}\right)^{\gamma} \\
& -\frac{2 \sigma(R)}{R}-\frac{4 \mu \dot{R}}{R}-4 \kappa_{s} \frac{\dot{R}}{R^{2}}-P_{0}-P(t) .
\end{aligned}
$$

The shell elasticity was modeled through a radiusdependent effective surface tension, for which the small oscillation amplitudes can be approximated by its first order Taylor expansion ${ }^{23}$

$$
\sigma(R)=\sigma\left(R_{0}\right)+2 \chi\left(\frac{R}{R_{0}}-1\right) .
$$

See Table I for a definition of the symbols used. Note that for $R_{c}=0$, Eq. (2) reduces to the classical RayleighPlesset equation of a coated gas bubble.

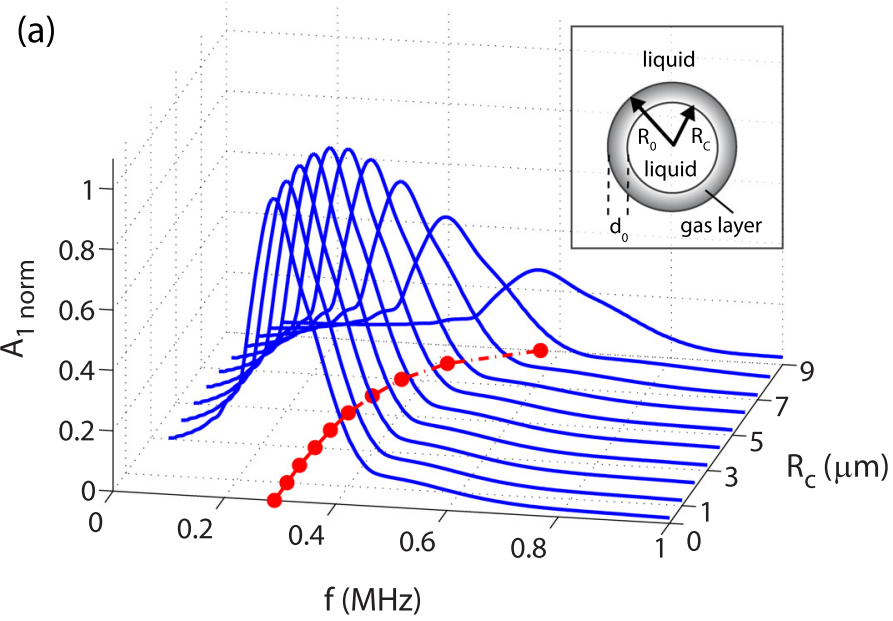

To investigate the resonance behavior of the model system described by Eq. (2), numerical simulations were performed. Initially, $\chi$ and $\kappa_{s}$ were set to 0 (i.e., representing an uncoated gas layer), and the surface tension $\sigma$ was set to $72 \mathrm{mN} / \mathrm{m}$, corresponding to the surface tension of a clean gas-water interface. The resting radius of the complex $R_{0}$ (with $R_{0}=R_{c}+d_{0}$, see the inset) was kept constant at $10 \mu \mathrm{m}$; the radius of the liquid core was varied between $R_{c}=0 \mu \mathrm{m}$, corresponding to a pure gas bubble, and $R_{c}=9 \mu \mathrm{m}$ with $1 \mu \mathrm{m}$ increments. The simulated resonance curves, normalized to the maximum response of the gas bubble, are shown in Fig. 3(a). It can be seen that increasing $R_{c}$ (decreasing the total gas volume) shifts the resonance peak to higher frequencies, as indicated by the red curve. The experimentally obtained resonance frequency of a StemBell of $10 \mu \mathrm{m}$ in the radius was found to be around $0.6 \mathrm{MHz}$ [see Fig. 2(b)], which is about twice the predicted resonance frequency of a similar sized pure gas bubble [see Fig. 3(a), for $R_{c}=0$ ]. Part of this $0.3 \mathrm{MHz}$ frequency shift can be explained by the lower amount of gas contained by a StemBell compared to a gas bubble. For example, in the case of the StemBell of Fig. 2(b), with $R_{c} \sim 8 \mu \mathrm{m}$ as determined by fluorescence microscopy, the modified equation (assuming an uncoated gas layer) predicts the resonance frequency to be at $0.42 \mathrm{MHz}$. The remaining part of the shift can be explained by the fact that the microbubbles surrounding the cell are coated with a lipid shell, thereby introducing some degree of elasticity, increasing the resonance frequency of the complex. In addition, we find friction within the system. We account for these effects via an effective shell elasticity $\chi$ and effective shell viscosity $\kappa_{s}$. By then, optimizing the fits between the experimental and simulated resonance curves, unique values for $\chi$ (affecting the resonance frequency) and $\kappa_{s}$ (affecting the width of the resonance curve) can be obtained. For the values of $\chi=1.5-3.0 \mathrm{~N} / \mathrm{m}$ and $\kappa_{s}$ of the order of $10^{-7} \mathrm{~kg} / \mathrm{s}$, the model predicts the resonance frequency of the StemBells with good overlap with the experimental resonance curves. A comparison between the experimental and simulated

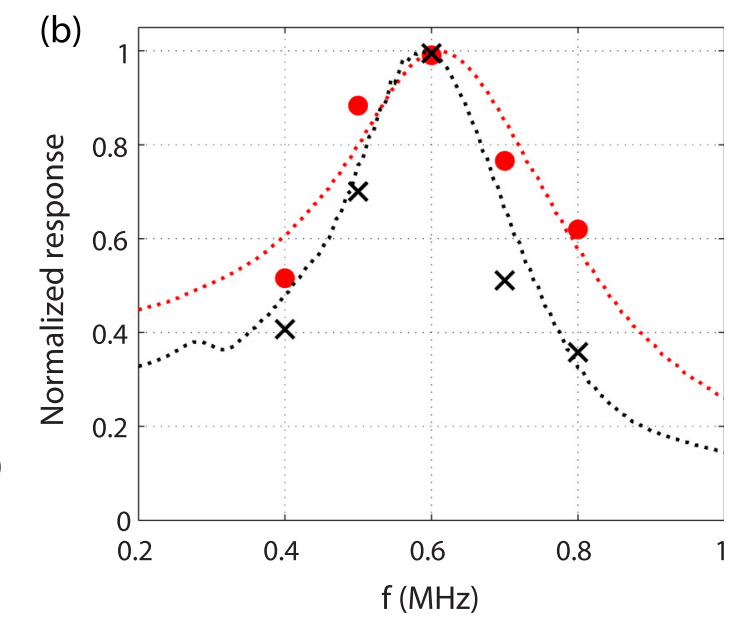

FIG. 3. (a) Simulated resonance curves of the uncoated model system (i.e., $\chi=0, \kappa_{s}=0$, and $\sigma=72 \mathrm{mN} / \mathrm{m}$ ) as a function of $\mathrm{R}_{c}$, while the total radius $R_{0}$ (see the inset) was set at $10 \mu \mathrm{m}$. The red curve shows resonance frequency versus $R_{c}$. (b) Comparison between experimental and simulated (dashed) resonance curves for two $10 \mu \mathrm{m}$ radius StemBells at $P_{-}=50 \mathrm{kPa}$. Good agreement with the experimental data was obtained for $\chi=1.5 \mathrm{~N} / \mathrm{m}$ and $\kappa_{s}=1 \times 10^{-7} \mathrm{~kg} / \mathrm{s}$ and (black) $\chi=2.8 \mathrm{~N} / \mathrm{m}$ and $\kappa_{s}=3 \times 10^{-7} \mathrm{~kg} / \mathrm{s}$ (red). The values of $R_{c}$ used in the simulations were set equal to the actual cell radii as determined by fluorescence microscopy: $6.7 \mu \mathrm{m}$ (red) and $8.1 \mu \mathrm{m}$ (black). The initial surface tension $\sigma\left(R_{0}\right)$ was set to $50 \mathrm{mN} / \mathrm{m}$. The curves are normalized to the maximum value of $A_{1}$. 
(a)

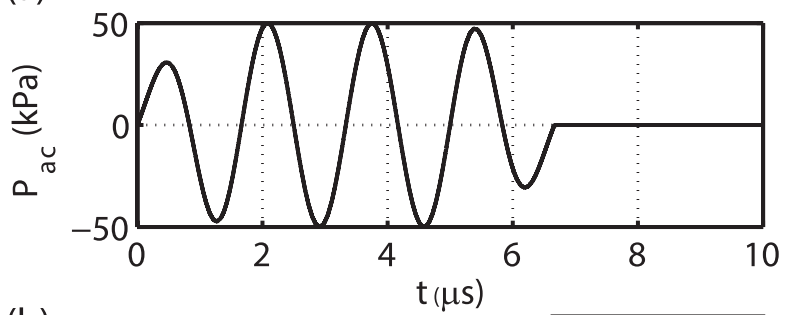

(b)

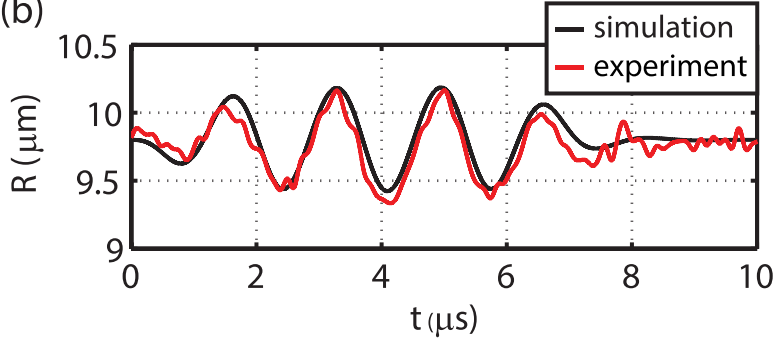

FIG. 4. (a) Simulated acoustical pressure wave of $P_{-}=50 \mathrm{kPa}$ and $f=0.6 \mathrm{MHz}$. (b) Comparison of an experimental $R(t)$-curve (red) and simulated $R(t)$-curve (black). The simulation parameters were $\kappa_{s}=8 \times 10^{-7} \mathrm{~kg} / \mathrm{s}$, $\chi=2.8 \mathrm{~N} / \mathrm{m}, \sigma\left(R_{0}\right)=50 \mathrm{mN} / \mathrm{m}$, and $R_{c}=6.6 \mu \mathrm{m}$. The resting radius of the StemBell was $9.8 \mu \mathrm{m}$.

(dashed) resonance curves of two StemBells (with $R_{0}=10 \mu \mathrm{m}$ ) is shown in Fig. 3(b).

Moreover, the model also gives a good estimate of the amplitude of oscillations of the StemBells for similar values of $\chi$ and $\kappa_{s}$. This is illustrated in Fig. 4. The black $R(t)$-curve in Fig. 4(b) represents the simulated response of a $9.8 \mu \mathrm{m}$ radius StemBell subjected to the 4 cycle ultrasound burst shown in Fig. 4(a) $\left(f=0.6 \mathrm{MHz}\right.$ and $\left.P_{-}=50 \mathrm{kPa}\right)$. The simulated dynamics are in good agreement with the experimental dynamics of a similar sized StemBell, represented by the red $R(t)$-curve. The parameter $R_{c}$ was set to $6.6 \mu \mathrm{m}$, which was obtained from the experimentally determined radius of the stem cell inside the StemBell.

In this paper, we have investigated the vibrational dynamics of StemBells during ultrasound exposure. We found that the relatively low resonance frequency of saturated StemBells $(\sim 0.6 \mathrm{MHz})$ is governed by the collective oscillations of the surrounding microbubbles. A modified Rayleigh-Plesset equation, considering the total gas volume of the surrounding layer of microbubbles, captured the resonance behavior and vibrational dynamics of the StemBells in detail. The results of this study will help to further optimize this radiation force-assisted stem cell delivery technique.

See supplementary material for a vibrating saturated StemBell in response to an 8 cycle ultrasound burst at $1 \mathrm{MHz}$ and $200 \mathrm{kPa}$. The video was recorded at 14.2 million frames per second (Mfps).

This work was financially supported by the Dutch Technology Foundation (STW). The authors would like to thank Frits Mastik, Robert Beurskens, Geert Springeling, and Michiel Manten for their technical assistance. The help of Dr. Klazina Kooiman is also appreciated. We also would like to thank Professor Andrea Prosperetti (Physics of Fluids Group, University of Twente) for the fruitful discussions related to the modeling of the StemBells.

${ }^{1}$ C. E. Murry, H. Reinecke, and L. M. Pabon, J. Am. Coll. Cardiol. 47, 1777 (2006)

${ }^{2}$ K. Malliaras and E. Marban, Br. Med. Bull. 98, 161 (2011).

${ }^{3}$ M. Hofmann, K. C. Wollert, G. P. Meyer, A. Menke, L. Arseniev, B. Hertenstein, A. Ganser, W. H. Knapp, and H. Drexler, Circulation 111, 2198 (2005).

${ }^{4}$ H. C. Quevedo, K. E. Hatzistergos, B. N. Oskouei, G. S. Feigenbaum, J. E. Rodriguez, D. Valdes, P. M. Pattany, J. P. Zambrano, Q. Hu, I. McNiece, A. W. Heldman, and J. M. Hare, Proc. Natl. Acad. Sci. U.S.A. 106, 14022 (2009).

${ }^{5}$ T. J. A. Kokhuis, I. Skachkov, B. A. Naaijkens, L. J. M. Juffermans, O. Kamp, K. Kooiman, A. F. W. van der Steen, M. Versluis, and N. de Jong, Biotechnol. Bioeng. 112, 220 (2015).

${ }^{6}$ L. Woudstra, P. Krijnen, S. Bogaards, E. Meinster, R. Emmens, T. J. A. Kokhuis, I. Bollen, H. Baltzer, S. Baart, R. Parbhudayal, M. Helder, V. van Hinsbergh, R. Musters, N. de Jong, O. Kamp, H. Niessen, A. van Dijk, and L. J. M. Juffermans, Stem Cell Res. 17, 6 (2016).

${ }^{7}$ P. Dayton, A. Klibanov, G. Brandenburger, and K. Ferrara, Ultrasound Med. Biol. 25, 1195 (1999).

${ }^{8}$ J. J. Rychak, A. L. Klibanov, K. F. Ley, and J. A. Hossack, Ultrasound Med. Biol. 33, 1132 (2007).

${ }^{9}$ P. J. Frinking, I. Tardy, M. Theraulaz, M. Arditi, J. Powers, S. Pochon, and F. Tranquart, Ultrasound Med. Biol. 38, 1460 (2012).

${ }^{10}$ T. Segers and M. Versluis, Lab Chip 14, 1705 (2014).

${ }^{11}$ P. A. Dayton, J. S. Allen, and K. W. Ferrara, J. Acoust. Soc. Am. 112, 2183 (2002).

${ }^{12}$ V. Garbin, D. Cojoc, E. Ferrari, E. Di Fabrizio, M. L. J. Overvelde, S. M. van der Meer, N. de Jong, D. Lohse, and M. Versluis, Appl. Phys. Lett. 90, 114103 (2007).

${ }^{13}$ B. A. Naaijkens, H. W. M. Niessen, H. J. Prins, P. A. J. Krijnen, T. J. A. Kokhuis, N. Jong, V. W. M. Hinsbergh, O. Kamp, M. N. Helder, R. J. P. Musters, A. Dijk, and L. J. M. Juffermans, Cell Tissue Res. 348, 119 (2012).

${ }^{14}$ A. L. Klibanov, P. T. Rasche, M. S. Hughes, J. K. Wojdyla, K. P. Galen, J. H. Wible, and G. H. Brandenburger, Invest. Radiol. 39, 187 (2004).

${ }^{15}$ T. J. A. Kokhuis, V. Garbin, K. Kooiman, B. A. Naaijkens, L. J. M. Juffermans, O. Kamp, A. F. W. van der Steen, M. Versluis, and N. de Jong, Ultrasound Med. Biol. 39, 490 (2013).

${ }^{16}$ J. R. Lindner, J. Song, J. Christiansen, A. L. Klibanov, F. Xu, and K. Ley, Circulation 104, 2107 (2001).

${ }^{17}$ C. T. Chin, C. Lancee, J. Borsboom, F. Mastik, M. E. Frijlink, N. de Jong, M. Versluis, and D. Lohse, Rev. Sci. Instrum. 74, 5026 (2003).

${ }^{18}$ E. C. Gelderblom, H. J. Vos, F. Mastik, T. Faez, Y. Luan, T. J. A. Kokhuis, A. F. W. v. d. Steen, D. Lohse, N. d. Jong, and M. Versluis, Rev. Sci. Instrum. 83, 103706 (2012).

${ }^{19}$ S. M. van der Meer, B. Dollet, M. M. Voormolen, C. T. Chin, A. Bouakaz, N. de Jong, M. Versluis, and D. Lohse, J. Acoust. Soc. Am. 121, 648 (2007).

${ }^{20}$ T. Leighton, The Acoustic Bubble (Academic Press, 1994).

${ }^{21}$ R. Omta, J. Acoust. Soc. Am. 82, 1018 (1987).

${ }^{22} \mathrm{~N}$. de Jong, A. Bouakaz, and P. Frinking, Echocardiography 19, 229 (2002).

${ }^{23}$ P. Marmottant, S. van der Meer, M. Emmer, M. Versluis, N. de Jong, S. Hilgenfeldt, and D. Lohse, J. Acoust. Soc. Am. 118, 3499 (2005). 